\title{
The Spatial Structure of Homogeneous Turbulence at High Reynolds Number
}

\author{
A. Vincent and M. Meneguzzi \\ CERFACS, 42 av. G. Coriolis, 31057 Toulouse, France
}

\begin{abstract}
A direct numerical simulation at resolution $240^{3}$ is used to obtain a statistically stationary three-dimensional homogeneous and isotropic turbulent field at a Reynolds number around $1000\left(R_{\lambda} \approx 150\right)$. The energy spectrum displays an inertial subrange extending over more than one decade. In the physical space, the strongest vorticity is organized in very elongated thin tubes. The typical length of a tube is around the integral scale of the flow and its thickness is a few dissipation scales.
\end{abstract}

In the past, direct numerical simulations of the three-dimensional Navier-Stokes equation have been restricted to modest Reynolds numbers (Orszag and Patterson (1972); Siggia and Patterson (1978); Siggia (1981)). These simulations have mostly given properties on the dissipation scale of a turbulent flow. It now becomes feasible, on computers of the latest generation, to reach Reynolds numbers at which there exists an inertial subrange in the energy spectrum (Kerr, 1985; Yamamoto and Hosokawa, 1988; She et al., 1988, 1990). The object of our work is to study a more extended inertial subrange.

We solve the Navier-Stokes equation for incompressible fluids in rotational form

$$
\frac{\partial v}{\partial t}=v \times \omega-\nabla\left(p+v^{2} / 2\right)+\nu \nabla^{2} v+f
$$

with the continuity equation $\nabla \cdot v=0, v$ is the velocity field, $\boldsymbol{\omega}=\nabla \times v$ the vorticity, $p$ the pressure, $\nu$ the kinematic viscosity and $f$ a force field. Since we are interested in (statistically) homogeneous turbulent flows, we take periodic boundary conditions in all directions, with period $2 \pi$. A pseudospectral method is used to compute the right-hand side of this equation (see Gottlieb and Orszag, 1977). The time marching is performed using a second order finite-difference scheme. We chose to force the field in a deterministic way, as was done by Kerr (1985). All Fourier modes with wavenumber components equal to 1 or 0 are forced with a constant amplitude $f$ independent of $k$.

The calculation presented here was done with $240^{3}$ Fourier components, and a viscosity of $10^{-3}$. This gives $R \approx 1000\left(R_{\lambda} \approx 150\right)$ (Vincent and Meneguzzi, $1990)$. After integration over a few tens of turnover times we obtain a statistically stationary regime, in the sense that the mean kinetic energy density fluctuates 
by a few percent around a constant value. Our time unit is such that the large eddy turnover time is 0.35 . The energy spectrum $E(k)$ at a given time is shown in Fig. 1 at time 14. A power law range can be seen for $k<25$, with an exponent a little larger than $-5 / 3$. The dashed line shows the same spectrum multiplied by $k^{5 / 3}$. The small increase in the energy spectrum at the highest wavenumbers is a numerical effect due to a lack of resolution of the very small scales in the dissipative range. It is not due to aliasing and does not affect the inertial range. We have redone a part of our runs with aliasing removed by the method of shifted grids described in Patterson and Orszag (1971), and we did not find any significant difference in the results. The fact that this is an inertial range is confirmed by inspection of the energy flux spectrum, shown in Fig. 2. The energy flux $\Phi(k)$, which is the rate of energy transferred per unit mass by all modes with $k^{\prime}<k$ to modes with $k^{\prime}>k$, is constant in the inertial range. This is seen in Fig. 2 to occupy a little more than one decade in wavenumber space.

A 3D view of the vorticity field is given in Fig. 3. The vorticity at each grid point is represented by a vector, here so small that individual vectors can hardly be seen. Vectors are only plotted if their modulus is larger than a given threshold. One can see that the vorticity is organized in thin elongated tubes, as previously reported by Siggia (1981), Kerr (1985) and She et al. (1990). The length of these tubes seems to be of order 1 , i.e. of the same order as the integral scale (the cube size is $2 \pi$ ). Their thickness is of the order of a few dissipation scales, here a few grid points. This is confirmed by a more detailed analysis, e.g. a cut through a typical vorticity tube, which shows clearly that its characteristic thickness is a few grid points. The dissipation scale, $l_{D}$, in our simulation is of the order of the mesh size, while the Taylor microscale is approximately ten mesh sizes. Therefore, the characteristic tube thickness seems to be intermediate between these two lengths.

From these visualizations, one is led to the conclusion that these vorticity tubes, which seem to be the basic structure of three-dimensional homogeneous turbulence, involve all the scales of the flow. We have done the same kind of pictures of the vorticity field after removing all dissipation range scales. The large scales to which the forcing is applied were also removed. One can see the external regions of the tubes. A single tube along with its environment has been extracted from the vorticity field by a pattern recognition technique (Fig. 4) (Villasenor and Vincent, 1990). Some helical structure can be seen, as noted by She et al. (1990). This might also correspond to secondary instabilities and is closely related to the dynamic of the tube itself.

Spatial correlations between vorticity and rate of strain were reported by Kerr (1985). At each grid point, we computed the eigenvalues $\lambda_{1}, \lambda_{2}, \lambda_{3}$ of the rate of strain tensor $S_{i j}=\frac{1}{2}\left(\partial_{i} v_{j}+\partial_{j} v_{i}\right) . \lambda_{1}$ is always negative and $\lambda_{3}$ is always positive $\left(\nabla \cdot v=0\right.$ implies $\left.\lambda_{1}+\lambda_{2}+\lambda_{3}=0\right)$. The intermediate eigenvalue $\lambda_{2}$ is found to be positive in approximately two thirds of the cases, in agreement with the results of the analysis of Kerr (1985) and Ashurst et al. (1987). Three-dimensional plots of the eigenvector fields are used to study the correlations with vorticity. Let us call $e_{1}, e_{2}, e_{3}$ the eigenvectors associated with $\lambda_{1}, \lambda_{2}, \lambda_{3}$ respectively, each eigenvector being given the amplitude and sign of its associated eigenvalue. The 
highest rate of strain is seen to be generally in the vicinity of vorticity tubes. The eigenvector $e_{3}$ associated with the positive eigenvalue, i.e. the stretching direction, is perpendicular to the vorticity. The eigenvector $e_{1}$, associated with the negative eigenvalue of the strain, is also perpendicular to the vorticity. The intermediate eigenvector $e_{2}$ is the one which is generally aligned with vorticity. Our conclusions are drawn from fully three-dimensional examination of the flow using rotation, zoom and clipping. We could not find an example of $e_{3}$ aligned with vorticity.

The general alignment of vorticity with $e_{2}$ reflects the quasi two-dimensionality of the vortex tubes. Indeed, in two dimensions, $\lambda_{2}=0, \lambda_{3}=-\lambda_{1}$ and $\omega$ would be perpendicular to the plane $\left(e_{1}, e_{3}\right)$. A discussion of this correlation of vorticity with the intermediate rate of strain direction is presented in Ashurst et al. (1987), where an explanation is proposed using a model due to Vieillefosse $(1982,1984)$. According to calculations using this model, a vorticity tube is created along the direction $e_{\mathbf{3}}$ of maximum stretching, but very soon becomes aligned with $e_{2}$. We are presently following vorticity tubes back in time until the time of their formation. Moreover, we observe that the maxima of velocity form a sheet perpendicular to the axis of the tube. In Fig. 5 the projection of the velocity field on a plane perpendicular to a vorticity tube is shown. Our preliminary impression is that the tubes are generated by an instability due to the shear associated with the velocity sheets. If this is the case, the above picture would not be correct. The results of this investigation will be presented in a forthcoming paper. Moreover, we observe that the large velocity regions are sheets located near and parallel to the axis of the tubes but directed perpendicularly to it.

Acknowledgements. The calculations presented were done on the Cray-2 of CCVR (Centre de Calcul Vectoriel pour la Recherche), Palaiseau, France.

\section{References}

Ashurst, W.T., Kerstein, A.R., Kerr, R.M., Gibson C.H.: 1987, Phys. Fluids 30, 2343 Gottlieb, D., Orszag, S.A.: 1977, SIAM CBMS-NSF Regional Conference Series in Applied Math. "Numerical Analysis of Spectral Methods."

Kerr, R.: 1985, J. Fluid Mech. 153, 31

Orszag, S.A., Patterson, G.S.: 1972, Phys. Rev. Lett. 28, 76

Patterson, G.S., Orszag, S.A.: 1971, Phys. Fluids. 14, 2538

She, Z.S., Jackson, E., Orszag S.A.: 1988, J. Sci. Computing 3, 407

She, Z.S., Jackson, E., Orszag S.A.: 1990, Nature 344, 226

Siggia, E.D., Patterson, G.S.: 1978, J. Fluid Mech. 86, 567

Siggia, E.D.: 1981, J. Fluid Mech. 107, 375

Yamamoto, K., Hosokawa, I.: 1988, J. Phys. Soc. Japan 57, 1532

Vieillefosse, P.: 1982, J. de Physique 43, 837

Vieillefosse, P.: 1984, Physica 125A, 150

Vincent, A.P., Meneguzzi, M.: 1990, to appear in J. Fluid Mech.

Villasenor, J.D., Vincent, A.P.: 1990, submitted to Computer Vision, Graphics, and Image Processing (submitted). 
A. Vincent and M. Meneguzzi

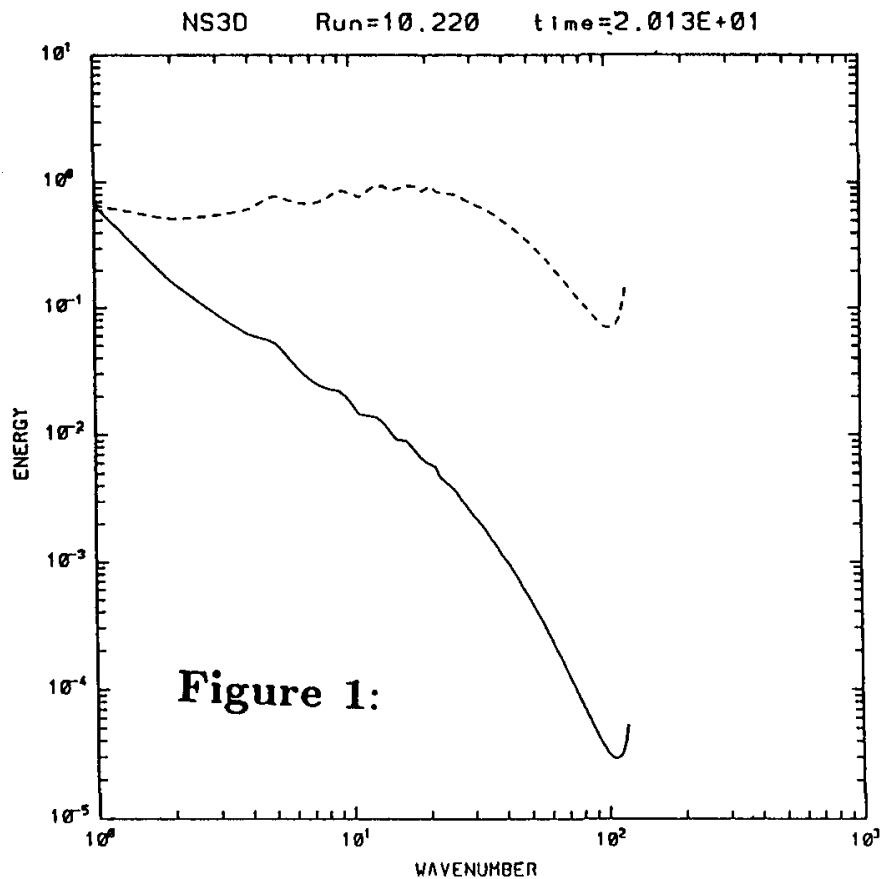

Fig. 1. Energy spectrum at a given time (continuous curve). Same energy spectrum multiplied by $k^{5 / 3}$ (dashed curve).

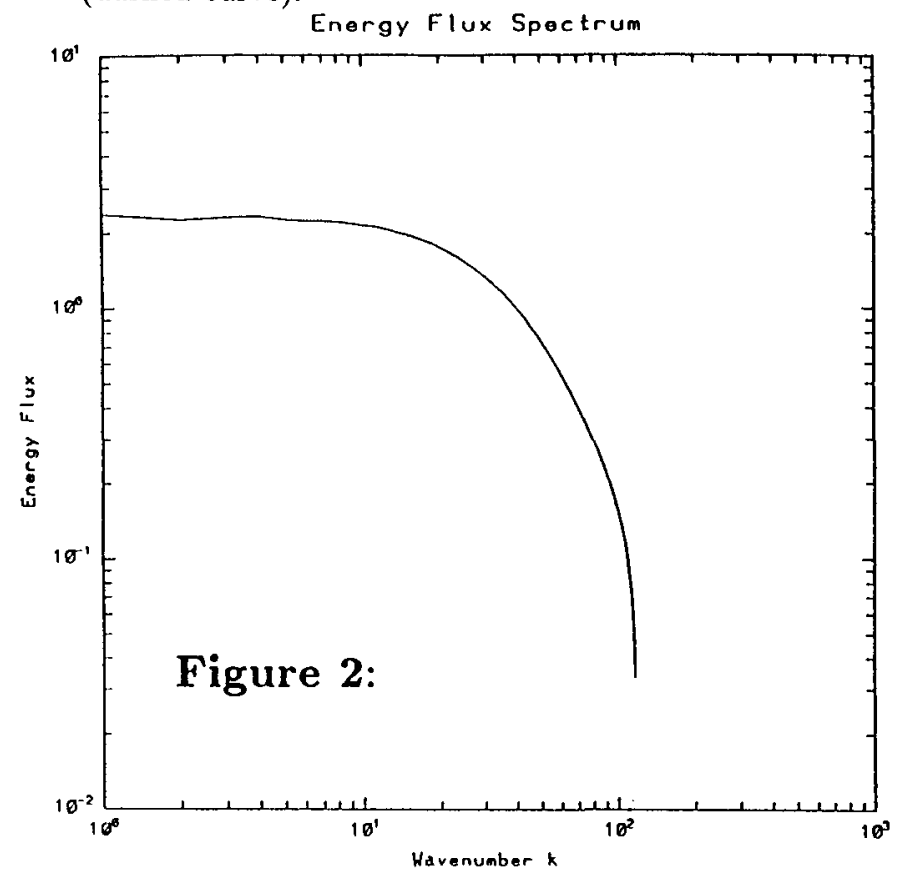

Fig. 2. Energy flux corresponding to the spectrum of Fig. 1. 
Figure 3:

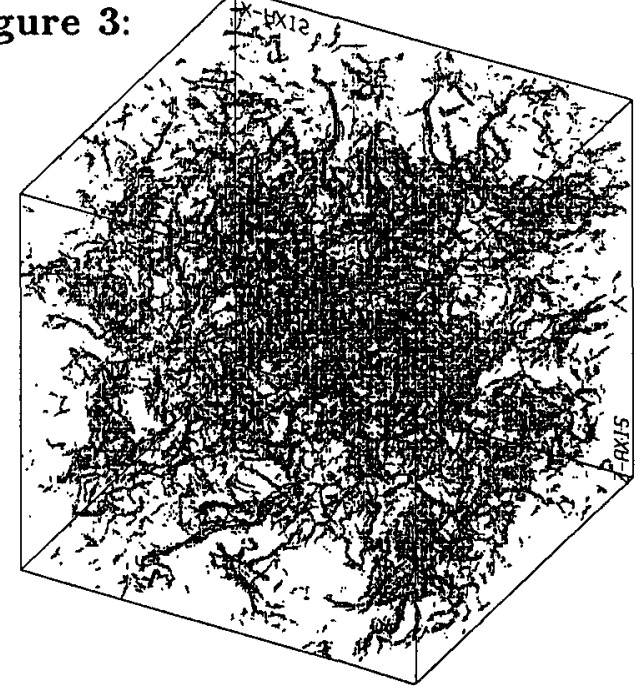

Fig. 3. View of the vorticity field, represented by a vector of length proportional to the vorticity amplitude at each grid point. Only vectors larger than a given threshold value are shown.

Fig. 4. A view of a single vorticity tube after it has been extracted by a pattern recognition technique.
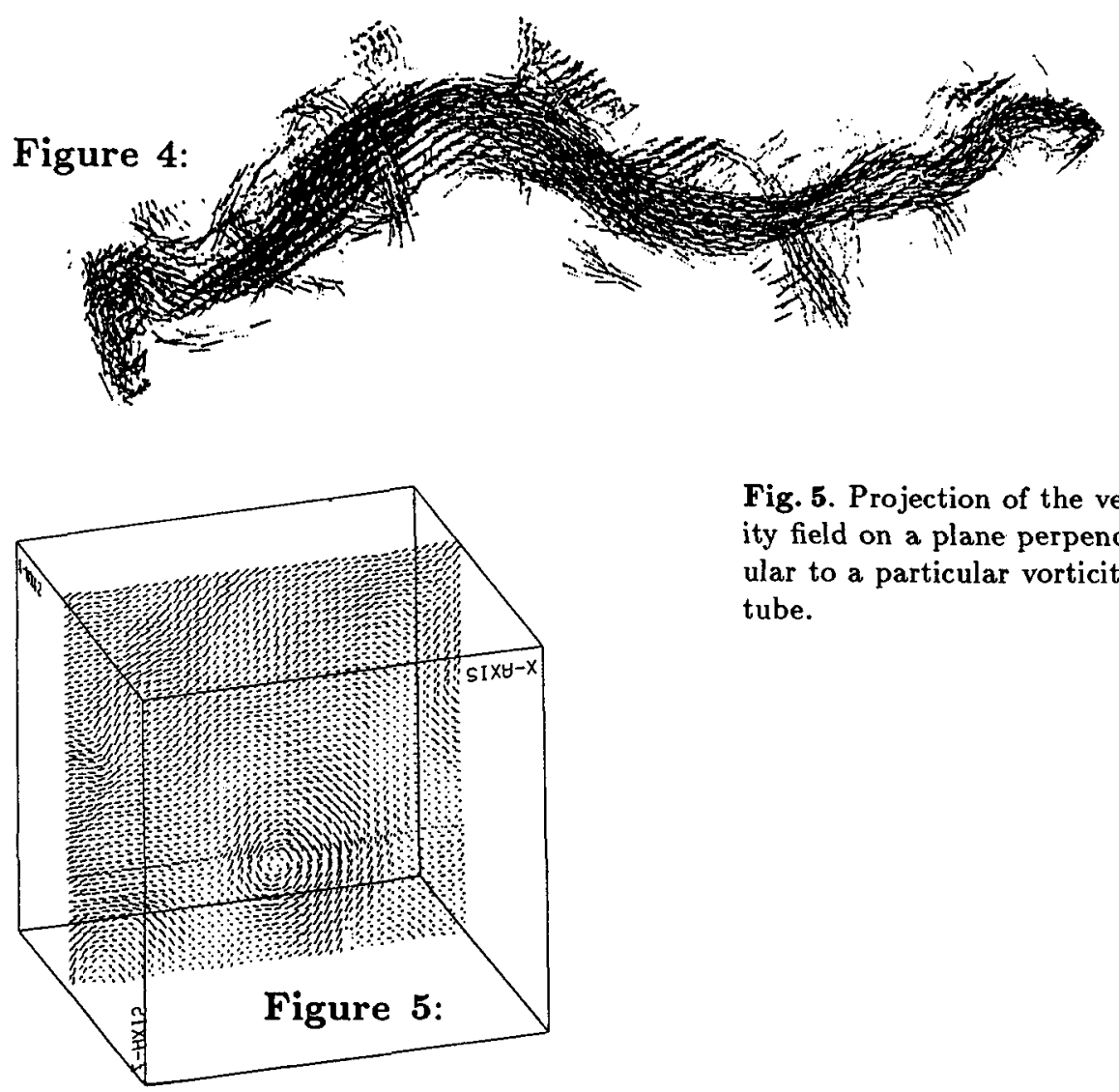

Fig. 5. Projection of the velocity field on a plane perpendicular to a particular vorticity tube. 